Théologiques

Théologiques

\title{
Pratiquer la justice sociale ou s'effondrer
}

L'actualité du message d'Amos (Am 5,7-13 ; 6,12)

\section{Nicodème Kolani Bakimani}

Volume 24, numéro 1, 2016

(In)justice sociale

URI : https://id.erudit.org/iderudit/1044737ar

DOI : https://doi.org/10.7202/1044737ar

Aller au sommaire du numéro

\section{Éditeur(s)}

Faculté de théologie et de sciences des religions, Université de Montréal

ISSN

1188-7109 (imprimé)

1492-1413 (numérique)

Découvrir la revue

Citer cet article

Kolani Bakimani, N. (2016). Pratiquer la justice sociale ou s'effondrer :

l'actualité du message d'Amos (Am 5,7-13 ; 6,12). Théologiques, 24(1), 15-36.

https://doi.org/10.7202/1044737ar
Résumé de l'article

De nos jours, la poussée des mouvements extrémistes et des violences liées à la précarité et aux inégalités pose avec plus d'acuité la question de la justice sociale. Il importe que les chrétiens et tous les hommes militant pour un avenir paisible, réentendent la voix des prophètes bibliques, lesquels ont dénoncé et présenté les injustices sociales comme facteurs de ruine. Dans cette perspective, il est urgent de réécouter plus particulièrement la voix d'Amos qui, dans l'histoire de la religion biblique, a su démontrer, pour la première fois, que les injustices sociales sont des actes insensés et autodestructeurs. Bien que proclamé il y a plus de 2500 ans, son message est d'actualité pour notre monde marqué par des tractations entre des débiteurs essoufflés des créanciers impitoyables vivant dans la surabondance ; il nous rappelle que toute société infestée par des injustices et de profondes inégalités sociales s'engage dans un processus de dé-création. 


\title{
Pratiquer la justice sociale ou s'effondrer
}

\author{
L'actualité du message d'Amos (Am 5,7-13; 6,12)
}

\author{
Nicodème Kolani Bakimani" \\ Exégèse biblique \\ Université de Strasbourg (France)
}

De nos jours où la précarité gagne du terrain même dans les pays développés et suscite des agitations et la percée de mouvements extrémistes et conservateurs, il importe pour les chrétiens et pour tous ceux qui veulent œuvrer pour un monde juste et apaisé, de réentendre la voix des prophètes bibliques qui sont incontestablement les premiers à avoir dénoncé et combattu les inégalités et les injustices qu'ils présentent comme une cause de la ruine sociale. Dans cette perspective, il convient de scruter plus particulièrement le message d'Amos, le précurseur des prophètes écrivains, celui qui, dans l'histoire de la religion biblique, a placé, pour la première fois, la pratique de la justice sociale comme la condition sine qua non pour que Dieu, créateur et maître de l'univers, puisse être présent dans et aux côtés de toute communauté croyante ou de tout groupe d'individus qui veulent vivre et prospérer. Il est intéressant de chercher à savoir quelle est l'actualité du message de ce prophète, le plus souvent cité lorsqu'il s'agit d'aborder les questions sociales. Quel héritage a-t-il légué aux divers acteurs, politicologues, philosophes, sociologues, artistes et autres personnes, qui, dans nos sociétés actuelles, marquées par des tractations entre des débiteurs de plus en plus essoufflés et des créanciers impitoyables et peu sou-

* Nicodème Kolani Bakimani est religieux franciscains, docteur en théologie et sciences religieuses de l'université de Strasbourg, spécialité théologie catholique, option exégèse biblique. Sa thèse de doctorat s'intitule: Étude de la place et de la fonction des supposés éléments tardifs du livre d'Amos. Une contribution à la lecture de type synchronique du livre d'Amos, thèse sur le livre du prophète Amos. Il est actuellement Secrétaire Général de l'Institut catholique missionnaire d'Abidjan (ICMA). Il a récemment publié (2016) "Amos, prophète et défenseur de la dignité humaine. Une lecture d'Am 2,6-16 à la lumière de la Déclaration universelle des droits de l'homme ", dans P. Keith, dir., Texture sacrée, l'intertexte biblique dans quelques ouvres littéraires et textes autorisés, Strasbourg, Presses Universitaires de Strasbourg, p. 59-92.

C Revue Théologiques 2016. Tout droit réservé. 
cieux de la misère des pauvres, veulent conscientiser nos contemporains quant à l'urgence et à la nécessité de la pratique de la justice sociale ? Une pléthore d'auteurs ${ }^{1}$ a déjà essayé d'actualiser son message: soit en l'appliquant à des situations précises d'injustice ou d'oppression de leur société (le plus souvent), soit en cherchant à déterminer la racine principale des exactions qu'ils dénoncent. Très récemment, Hahling, après avoir établi un rapport entre l'époque d'Amos et la nôtre, a démontré que sa prédication reste très pertinente pour notre époque, en ce qu'elle révèle que, "plus un peuple s'éloigne de Dieu, plus il enfreint ses règles dont celle de s'occuper des pauvres» (Hahling 2013, 132). Sans nier cette importante dimension, nous nous intéresserons, dans le présent article, à la manière dont ce prophète présente les injustices sociales et leurs conséquences sur le devenir d'Israël, sa terre de mission, afin de susciter un changement d'attitude chez leurs auteurs. Plus concrètement, nous poserons que la pérennité du message d'Amos et son actualité résident en ce qu'il montre que les injustices sont des actes de dé-création et qu'Israël et, par-delà toute société, qui n'est point bâtie sur la justice et sur le droit, est vouée à périr.

Cependant, dans cette étude, il ne nous sera pas possible de procéder à une analyse exhaustive de toute la prédication d'Amos. Aussi, nous semble-t-il judicieux d'examiner Am 5,7-13 et Am 6,12, deux passages clefs qui apparaissent souvent dans les discussions sur la critique sociale d'Amos, passages dont le contenu démontre clairement que toute perversion de la justice et du droit est contraire au projet divin et, de ce fait, est un facteur de désordre et de ruine. Notre article s'articulera autour de trois points, suivis d'une conclusion dans laquelle nous soulignerons le bien fondé du message d'Amos pour notre monde actuel. Tout d'abord, nous rappellerons très brièvement le contexte socio-économique d'Israël afin de faire ressortir les raisons profondes qui ont suscité l'intervention inattendue

1. Il serait impossible de présenter ici une liste exhaustive de tous ceux qui ont travaillé sur Amos et la justice sociale. Voir à ce propos, outre les auteurs que nous citerons dans cet article, les récents ouvrages faisant l'état de la recherche du livre d'Amos (Melungin 1998, 65-101; Carroll 2002, 127-131). Martin-Achard (1984, 162-271) retrace aussi l'histoire de l'exégèse du livre d'Amos et montre que sa critique sociale a été très utilisée par les réformateurs, les adeptes de la théologie de la libération et d'autres penseurs modernes. De même, Hasel (1991, 101-104), présente également les différentes approches de la critique sociale d'Amos dès les années 1950 à nos jours.

2. Asurmendi $(1988,15)$ situe aussi la racine profonde des injustices dénoncées par Amos dans l'oubli de Dieu. À propos de l'orgueil reproché aux chefs militaires en Am 6,1-3.13-14; 7,9, il écrit: "séparé de la base de leur existence, ils oppriment leurs frères [...]. L'oubli de Dieu conduit à l'élimination de l'autre». 
d'Amos puis, dans les deux points suivants, nous analyserons respectivement Am 5,7-13 et Am 6,12 dans le but de montrer qu'Amos présente essentiellement les injustices sociales comme des facteurs de ruines, des actes déraisonnables et autodestructeurs.

\section{Le climat politique et socio-économique d'Israël à l'époque d'Amos}

Comme l'indique l'en-tête de son livre, Amos, l'un des bergers de Teqoa - une bourgade située à une douzaine de kilomètres au sud-est de Bethléem et à moins de dix-neuf kilomètres de Jérusalem — a prophétisé contre Israël sous le règne de Jéroboam II (786-746) et d'Ozias, roi de Juda (783-742), son pays d'origine (Am 1,1-2). En tenant compte de la durée du règne de Jéroboam II et d'autres données du livre, telle la mention "deux ans avant le tremblement de terre ${ }^{3}$ » $(\mathrm{Am} 1,1)$, certains exégètes et historiens situent son intervention autour de 760-750 avant notre ère. Même s'il leur est difficile de déterminer avec précision le nombre d'années ou la durée exacte de sa prédication ${ }^{4}$, généralement les spécialistes s'accordent sur les caractéristiques majeures de cette période.

Les chercheurs soulignent habituellement que c'était une période durant laquelle Israël connaissait la paix dans ses frontières. En effet, les Israélites avaient profité de l'abaissement momentané de la Syrie, leur ennemi le plus redoutable du nord, pour reconquérir Lodevar et Quarnaïm - deux territoires transjordaniens (Am 6,13-14; 2 R13, 25; 14,25-28) — et étendre ainsi leurs possessions jusqu'aux limites de l'ancien empire davidique, asseyant une stabilité politique durable dans leur pays (Lemaire 1981, 45-48).

L'expansion des frontières, la stabilité politique et la paix retrouvée avaient favorisé le développement de l'agriculture et surtout la floraison du commerce extérieur, lequel avait généré beaucoup de richesses et donné naissance à une forme de capitalisme urbain. Les habitants cherchaient à tirer le maximum de profit des terres, de l'artisanat et du commerce. Les taxes sur les frontières et à l'intérieur du royaume furent alors renforcées,

3. Il n'y a certes pas d'unanimité sur la datation de ce tremblement de terre évoqué par le prophète Zacharie (Za 14,5) et par Flavius Joseph dans ses Antiquité Juives. Mais la plupart des biblistes et des historiens s'appuient souvent sur l'évocation de l'éclipse de soleil en Am 8,9, éclipse que certains textes assyriens et des calculs astronomiques situent au 15 juin 763, et surtout sur les résultats des fouilles archéologiques menées en 1956 par Yadin et son équipe à Hasor, pour le dater entre 765-760 avant notre ère (Wolff 1977, 124; Vesco 1980, 181).

4. Martin-Achard (1984, 38-45) fournit un bon résumé des différentes hypothèses concernant la durée exacte de la prédication d'Amos. 
mais au profit de la monarchie et de ses hauts fonctionnaires (Neher 1982, 445; da Silva 1997, 10-12). D'ailleurs, le livre d'Amos regorge de nombreux indices attestant qu'Israël connaissait une situation extrêmement prospère au moment de l'intervention d'Amos. À titre d'exemples, le prophète parle des maisons d'été et d'hiver (Am 3,15), des maisons bâties en pierre de taille (Am 5,11), des palais ornés de mobiliers en ivoire (Am $3,15 ; 6,4)$, des banquets somptueux et fréquents au cours desquels des boisons raffinées, de jeunes béliers et des veaux sont consommés (Am 6,4-6). Il évoque également un culte extrêmement brillant avec une grande assistance et de nombreux pèlerinages dans les sanctuaires royaux, comme Béthel (Am 8,5; 4,4; 5,5; 7,10), Dan (Am 8,14) et Gilgal (Am 4,4; 5,5).

Cependant, en accord avec plusieurs autres données du livre, des biblistes tels Vesco (1980, 482-483), Schottroff $(1984,35)$ et Halhling (2013, 22-25) relèvent également que les retombées de cette croissance économique ne profitaient malheureusement pas à tous les fils d'Israël. Aussi, sur le plan social, la société était-elle marquée par un très profond clivage. À côté de la classe des nantis, qui baignait dans un luxe exubérant et cherchait à accroître toujours davantage sa fortune par tous les moyens, il y avait la classe des nécessiteux désignés tout au long du livre par quatre qualificatifs très significatifs (Am 2,6-8; 4,1; 5,11-12; 8,4-6): 'ébyōn (le pauvre ou l'indigent ${ }^{5}$ ), dallîm (les faibles ${ }^{6}$ ), 'anāwîm (les humbles ${ }^{7}$ ), șaddîq $\left(\right.$ le juste $^{8}$ ). En parcourant certains oracles (Am 2,6-8; 3,10-15; 5, 7-12; $8,4-14)$, nous découvrons que, derrière ces débordements de richesse, se cache une toute autre réalité sociale. Les membres de la famille royale, les fonctionnaires de la cour, les militaires (Am 3,9-10; 6,1-3.13-14; 7,9), les riches et leurs femmes (Am 4,1-3), les juges (Am 6,7.10-12), les commerçants (Am 8,4-8) et les prêtres (Am 7,10-17), qui appartenaient à la classe opulente, vivaient dans l'insouciance et dans l'euphorie, et abusaient de

5. Ce substantif désigne une personne indigente, un miséreux ou un indigent (Botterweck 1974, 31-32).

6. Substantif pluriel de dal, qui signifie «chétif» ou «maigre» (Gn 41,19; Jg 6,15; Pr 19,7), ce terme désigne des personnes impuissantes, désarmées et livrées entièrement à la merci des puissants qui les oppriment et les écrasent (Fabry 1978, 228-230; Koehler et al. 1994, 221).

7. Dans la prédication d'Amos, ce mot désigne des personnes humiliées qui supportent dignement et consciemment les injustices dont elles sont victimes (Asurmendi 1988, 13).

8. Ce substantif désigne, dans la bouche d'Amos, l'innocent, c'est-à-dire celui qui est maltraité et qui souffre sans qu'il y ait le moindre motif même apparent ou formel (Botterweck 1974, 31-32). 
leur pouvoir en opprimant et en exploitant les pauvres. Ces fortunés, nonchalants, allongés sur des lits d'ivoire ou au creux des divans meublant leur maison en pierre de taille (Am 3,10-15; 5,11), buvaient du vin raffiné dans de larges coupes, se régalaient de la chair de jeunes béliers et de veaux, dansaient à pas improvisés lors de grands festins organisés régulièrement $(6,4-6)$. Sous les ordres de leurs femmes qui, sans cesse, leur réclamaient à boire (Am 4,1), ils opprimaient les pauvres, en saisissant impitoyablement leur part de grain (Am 5,11-12).

Quant aux pauvres, non seulement ils se voyaient injustement dépossédés de leurs moindres biens (Am 2,7-8; 5,10-12), mais ils étaient aussi vendus pour une somme d'argent modique ou troqués contre une paire de sandales $($ Am 2,6). En outre, tout recours à la justice leur était fatal en raison de la corruption des tribunaux, lesquels n'hésitaient pas à condamner l'innocent, en acquittant le coupable (Am 2,7; 6,10-12).

Nous retenons de ce qui précède qu'Israël, au moment de l'intervention d'Amos, était certes une nation prospère et apparemment paisible mais elle était infestée de l'intérieur par des injustices graves, touchant à toutes les structures sociales: sur la place du marché (Am 2,6; 8,4-6), dans les tribunaux (Am 2,6-7; 5,7.10-12), dans la famille [des filles domestiques réduites à l'esclavage sexuel $(\mathrm{Am} 2,7 \mathrm{~b})^{9}$ ], et même dans les lieux de culte $($ Am 2,$8 ; 8,4)$. Aussi, Amos est-il intervenu sur ce territoire ${ }^{10}$ non pas en

9. Barstad (1984, 33-36), Bentley (2006, 30), Garett (2008, 61) et d'autres défendent qu'en Am 2,7b il est question de prostitution sacrée. D'autres biblistes estiment que hanna ‘ ărāh désigne une divinité païenne et en déduisent qu'Amos dénonce l'idolâtrie (Soggin 1987, 48; Andersen et Freedman 2008, 318). Mais maints auteurs, comme entre autres, Amsler (1982) et Moughtin-Mumby (2011, 61-82), donnent des raisons suffisantes permettant de croire qu'il s'agit d'une domestique abusée sexuellement par un homme et son père. Ils relèvent précisément que nul ne peut soutenir qu'il s'agit d'une prostituée quelconque ou sacrée sans isoler le crime d'Am 2,7b des autres exactions dénoncées par Amos et sans faire de ce verset un corps étranger, n'ayant aucun lien logique avec l'ensemble formé par Am 2,6b-8, voire avec tout le reste du livre, centré sur la justice sociale.

10. Les motifs de l'intervention d'Amos sont diversement perçus. Certains auteurs, tel Renan (1889, 425-426), ont jadis présenté Amos comme un pauvre et un envieux de la richesse des riches qui a quitté son royaume pour venir s'en prendre aux fortunés du nord. D'autres penseurs modernes l'ont parfois taxé d'être «le premier marxiste avant Marx» (Loretz 1975, 272). D'autres encore le prennent pour un nostalgique de la vie rurale critiquant le capitalisme, le nouvel ordre social en expansion dans le Royaume du Nord. Ils perçoivent souvent sa critique sociale comme une dénonciation de la richesse et surtout du capitalisme foncier (Wanke 1972, 10; Lang 1982, 49). Mais de telles opinions ont du mal à s'imposer chez les chercheurs persuadés qu'Amos ne condamne pas la richesse en soi et ne défend pas la pauvreté comme un 
raison de sa grande prospérité économique mais essentiellement à cause de son système social bâti sur la paupérisation et les injustices canonisées comme un moyen de gain ${ }^{11}$. Dieu l'a brusquement arraché de derrière son troupeau et l'a envoyé $(\mathrm{Am} 7,14)$ dire à ceux qui commettent les injustices, qu'ils sapent les fondements de leur pays et l'entraînent vers une ruine inéluctable.

Après cette brève présentation du contexte sociopolitique d'Israël, nous procédons à l'analyse successive d'Am 5,7-13 et d'Am 6,12 afin de démontrer qu'Amos, dans sa critique sociale, présente les injustices comme des actes stupides et autodestructeurs dans le but d'amener ceux qui les légitimaient pour accroître leur fortune, à opérer une conversion radicale et immédiate pour éviter la chute de leur nation, Israël.

\section{Les injustices sociales, un facteur de ruine et de dé-création (Am 5,7-12)}

Avant d'étudier le contenu d'Am 5,7-13, un passage dont l'unité suscite souvent des prises de positions divergentes ${ }^{12}$, nous le situons dans son contexte littéraire. Il fait partie du cinquième chapitre du livre (Am 5,127), plus précisément de sa première sous-section (Am 5,1-17), laquelle, depuis les travaux de Waard $(1977,170-177)^{13}$, est souvent considérée comme une unité composée de divers éléments de genre et de style différents $^{14}$, disposés de façon concentrique ou chiastique. En amont, il est

idéal (Huffmon 1983, 114 ; Hasel 1991, 102-103). La richesse qu'Amos dénonce est essentiellement celle acquise à coup de graves injustices.

11. Il est utile de souligner avec Schottroff $(1984,35)$ qu'Amos ne dépeint presque jamais la misère des pauvres, ni ne dénonce la richesse des fortunés en soi; il rejette le fait que cette richesse est le fruit de la corruption et de l'oppression des pauvres.

12. Les v. 8-9 sont un fragment d'hymne et leur contenu semble, à premier vue, très différent de celui des v. 7.10-13. Pour cette raison, les exégètes estiment souvent que ces versets ne sont pas à leur place parce qu'ils empêchent les v. 10-13 de suivre directement le v. 7, verset avec lequel ils entretiennent un lien thématique (Amsler 1982, 210-211; Vesco 1980, 497). Mais cette position qui a longtemps dominé l'histoire de l'exégèse du livre d'Amos est de plus en plus contestée par un certain nombre de commentateurs récents (Story 1980, 67-80; Pfeiffer 1991, 475-481). Ce débat importe très peu pour cet article. Notre choix est de lire ce passage tel que la tradition nous l'a transmis sans pour autant insinuer que le corpus amosien a été rédigé en un seul jet.

13. Depuis Waard, beaucoup d'exégètes soutiennent qu'Am 5,1-17 se présente comme un ensemble organisé de façon concentrique. Mais ils ne s'accordent pas sur l'élément central de cette structure (Bovati et Meynet 1994, 169).

14. On y dénombre un chant funèbre formulé avec des verbes au parfait-passé (Am 5,23), une invitation pressante à chercher le Seigneur (Am 5,4-6), une dénonciation du 
précédé par une qinah, c'est-à-dire, un chant funèbre dans lequel le prophète se lamente sur la chute d'Israël (Am 5,1-3) et, plus immédiatement, par une invitation pressante faite aux acteurs des injustices à chercher Dieu afin d'éviter leur anéantissement (Am 5,4-6). En aval, il est suivi d'une nouvelle invitation dans laquelle le prophète intime prestement l'ordre aux mêmes coupables d'exactions exposées en Am 5,7.10-12 de chercher Dieu en rétablissant le droit et la justice au tribunal (Am 5,14-15); celle-ci débouche sur l'annonce de la destruction d'Israël comme imminente et inévitable (Am 5,16-17). Ces deux invitations pressantes (Am 5, 4-6; 5, 14-15), encadrant Am 5,7-13, permettent de déduire que les injustices sociales dénoncées dans ce passage constituent la cause profonde de la chute d'Israël, objet de la lamentation d'Am 5,1-3 et de l'annonce d'Am 5,16-17 (Story 1980, 67-80).

Nous poursuivons l'étude d'Am 5, 7-13 en procédant à la traduction de son texte hébraïque.

\section{Traduction d'Am 7, 5-13}

\begin{tabular}{|c|c|}
\hline${ }^{7}$ Hahōp̄okîm lalaৎănāh mišpāt & Ils changent le droit en poison ${ }^{15}$ \\
\hline ūṣadāqāh lā ’āreș hinnîhūu. & et jettent la justice par terre. \\
\hline 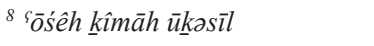 & Celui qui crée les pléiades et Orion, \\
\hline wahōpêk labbōqer salamāwet & change la nuit obscure en clarté matinale, \\
\hline weyōwm laylāh heḩ⿰š̀ik & réduit le jour en une nuit obscure \\
\hline haqqōwrê lamê-hayyam & convoque $^{16}$ les eaux des mers \\
\hline wayyišapə kêm 'al pənê hāāāreș & pour les répandre sur la surface de la terre, \\
\hline yəhwāh šamōw. & il se nomme le Seigneur. \\
\hline
\end{tabular}

renversement de la justice et du droit (Am 5,7), une doxologie exaltant les actions du Seigneur (Am 5,8-9), une dénonciation de l'exécration de celui qui réprouve la perversion de la justice (Am 5,10-13), une nouvelle invitation impérative à chercher le Seigneur (Am 5,14-15) et une annonce de malheur (Am 5,16-17).

15. Le terme $l a^{\varsigma} a \breve{n} \bar{a} h$ que nous avons rendu par "poison» désigne littéralement l'absinthe, plante amère qui, dans la littérature biblique, est habituellement assimilée au poison (Dt 29, 17; Jr 9,14; 23,15; Pr 5,4; Lm 3,15.19; Sir 31,49).

16. Le terme haqqōwrê est le participe présent masculin singulier du verbe qârâ, qui signifie «appeler quelqu'un par son nom » (Gn 27,1;39,15) ou «inviter quelqu'un » (1 S 9,13; $1 \mathrm{R} \mathrm{1,9)} \mathrm{ou} \mathrm{encore} \mathrm{"convoquer} \mathrm{une} \mathrm{personne} \mathrm{",} \mathrm{c'est-à-dire,} \mathrm{lui} \mathrm{intimer}$ l'ordre de se présenter devant soi (Gn 12,18). Ce troisième sens permet de garder l'idée du commandement que dénote l'action décrite par ce mot. 


\begin{tabular}{|c|c|}
\hline${ }^{9}$ Hammabolîīg šo $\underline{d}{ }^{\varsigma} a l-{ }^{\varsigma} a z$ & Il fait surgir ${ }^{17}$ la dévastation ${ }^{18}$ sur l'homme fort \\
\hline wəšs̄ód mīboșār yāb $\underline{b} \bar{o} w^{?}$. & et la dévastation entre dans la citadelle. \\
\hline 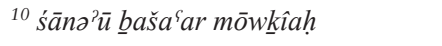 & Ils haïssent celui qui rappelle à l'ordre au tribunal ${ }^{19}$ \\
\hline 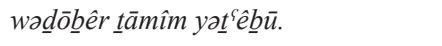 & et exècrent celui qui parle avec intégrité. \\
\hline${ }^{11}$ Lākêen yāa an bōwōšasakem ' $a l-d \bar{a} l$ & Aussi, parce que vous pressurez l'indigent, \\
\hline $\bar{u} m a s^{2} a \underline{t}$ bar tiqḥ̂u mimmennū & en lui prenant sa part de grain, \\
\hline battē ḡāzît banîtem & les maisons en pierres de taille que vous avez bâties \\
\hline walō têšabū $\underline{b} \bar{a} m$ & vous n’y habiterez pas, \\
\hline karamê hemed notaşatem & les vignes de délices que vous avez plantées, \\
\hline walō tišstū 'ete-yênām. & vous ne boirez pas leur vin. \\
\hline${ }^{12}$ Ki yad $a^{\varsigma}$ tî rabbîm pišs enkem $^{2}$ & Car je sais qu'elles sont nombreuses vos rebellions \\
\hline wa'asumîm hatțōtêkem & et monstrueux vos péchés, \\
\hline sorarê saddiq & oppresseurs du juste, \\
\hline lōqahê $\underline{k} \overline{o p e r}$ & extorqueurs de rançons \\
\hline wə’ebəyōwnîm baššs $a^{\varsigma} a r$ hițtū. & vous qui déboutez les pauvres du tribunal. \\
\hline${ }^{13}$ Lākên hammaškîl bāe $\underline{e} \underline{t}$ hahî yiddōm & c'est pourquoi l'homme prudent en ce temps-ci se tait \\
\hline$\underline{k} \hat{\imath} \varsigma^{\varsigma} \underline{t} r \bar{a}^{\varsigma} \bar{a} h h \hat{\imath}^{?}$ & Car c'est un temps de malheur. \\
\hline
\end{tabular}

17. Le mot Hammabalîg que nous avons traduit par «fait surgir» est fortement discuté parce qu'il n'est employé nulle part ailleurs dans le livre et que son étymologie et ses quelques rares occurrences dans d'autres livres bibliques n'aident pas à rendre compte de son sens contextuel. D'après van Hoonacker (1908, 241), ce terme serait issu d'un mot arabe qui signifierait «briller» ou «luire» et qui serait généralement utilisé pour décrire l'aurore. Il est le participe présent du hifil du verbe $\underline{b} \bar{a} l a \bar{g}$, attesté dans le Ps 39, 14 et dans Jb 9, 27; 10, 20 où il signifie « réjouir ». Mais il n'y a aucun sens à dire que Dieu «fait briller» ou «fait réjouir» la destruction sur l'homme fort. Les commentateurs, soucieux de préserver l'idée de l'éclat et de la soudaineté qu'il dénote, rendent ce mot par «faire surgir» ou par "déchaîner» (Bovati et Meynet 1994, 165-166; Andersen et Freedman 2008, 486).

18. Le substantif $\check{s} \bar{o} \underline{d}$, employé en Am 3,10 comme synonyme de violence (oppression), est abondamment utilisé dans d'autres textes prophétiques ( 11 fois) pour désigner une violente action ou une dévastation (Is 13,6; 16,4; 51,19; Jr 6,7; 48,3; Os 7,13; $9,6)$. Il est donc préférable de le rendre par «destruction» ou par «dévastation» (Freedman et Welch 2004, 412-418).

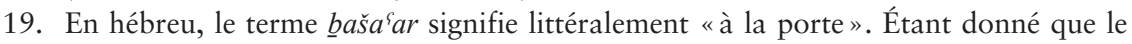
lieu faisait office du tribunal à l'époque d'Amos, nous avons préféré nous inscrire dans la perspective des biblistes tels les traducteurs de la TOB qui rendent ce substantif par «tribunal». 
Après la traduction d'Am 5,7-13, nous continuons son analyse en dégageant une structure opératoire dévoilant l'articulation de son contenu.

\section{La structure et le contenu d'Am 5,7-13}

La question de l'organisation d'Am 5,7-13 suscite des prises de positions divergentes ${ }^{20}$. Cette divergence résulte essentiellement des critères de structuration mis en exergue par chaque commentateur. Sans entrer dans ce débat récurrent, centré avant tout sur les thèmes majeurs de ce passage, nous proposons une structuration basée sur les différents acteurs dont les actions ou les attitudes sont rapportées par le narrateur. En effet, une analyse minutieuse tenant compte des deux différents protagonistes, Dieu qui parle par son prophète et les auteurs des injustices (coupables), nous permet de discerner, en Am 5,7-12, une structure opératoire très significative. Sans aucune ambigüité, il peut être vu comme un passage exposant des actions de deux acteurs différents [celles des coupables (v. 7.10-12) et celles de Dieu (v. 8-9)] et l'attitude de l'homme sensé, qui se tait (Am 5,13). Plus précisément, nous relevons tout d'abord deux actions des coupables portant successivement sur le droit et la justice (v. 7), situées en amont de cinq actions de Dieu énumérées dans les v. 8-9, puis, sept ${ }^{21}$ autres actions de ces mêmes coupables concernant les prophètes, l'indigent, les maisons, les vignes, le juste, les pauvres, exposées en aval de celles de Dieu (Am 5,10-12). Ainsi, nous pouvons représenter l'organisation de l'énoncé d'Am 5,7-13 sous la forme du schéma suivant:

A Les actions des coupables: ils changent le droit en poison et traînent la justice par terre (Am 5,7).

B Les actions de Dieu: il crée les Pléiades et l'Orion, change l'obscurité en clarté matinale, réduit le jour en sombre nuit, convoque les eaux pour les répandre sur la terre, livre l'homme fort à la dévastation (Am $5,8-9)$.

20. Bovati et Meynet (1994, 168-169) y voient une unité composée de cinq parties, disposées de façon concentrique. Sa structure qui a inspiré celle de Rottzoll $(1996,3)$ et qui ne discerne que trois séquences (Am 5,$7 ; 5,8 ; 5,9-13)$ a été très remise en cause par Möller (2003, 71), qui la juge trop forcée.

21. Cette liste ne tient compte que des verbes ayant pour sujet les coupables désignés tantôt par la troisième personne du pluriel («ils») ou par la deuxième personne du pluriel ("vous») ainsi que les qualificatifs qui leur sont donnés. Les verbes yā̌š (résider) et šătāh (boire) ne sont pas pris en compte parce qu'ils décrivent des actions que les auteurs d'injustice ne pourront plus accomplir. 
A' Les actions des coupables: ils haïssent celui qui rappelle à l'ordre le tribunal, abominent celui qui parle avec intégrité, pressurent l'indigent en lui saisissant sa part de grain, bâtissent des maisons en pierre, plantent des vignes de délices, oppressent le juste, extorquent les rançons, déboutent le pauvre au tribunal (Am 5,10-12).

C L'attitude de l'homme avisé: il se tait parce qu'il perçoit un temps de malheur (Am 5,13).

Tout d'abord, cette structure révèle un parallélisme entre A (v. 7) et A' (v. 10-12) parce qu'ils ont les mêmes acteurs et que leurs actions sont toutes des crimes contre la justice. En ce sens, nous soulignons que l'accusation d'Am 5,7 trouve son prolongement en celui d'Am 5,10-12 où sont énumérées sept actes concrets dont cinq, situés dans le présent, connotent la perversion de la justice et du droit, et deux, dans le passé, dévoilent à la fois l'identité des coupables et quelques motifs de leurs comportements. En effet, Am 5,11-12 nous renseigne sur le fait que ce sont les propriétaires des maisons en pierre et des vignes de délice $(\mathrm{Am} 5,11)$ qui changent le droit en poison et jettent la justice par terre (Am 5,7). Il nous apprend également que c'est en raison de telles réalisations qu'ils réduisent au silence le prophète ${ }^{22}$ qui dénonce le renversement du droit et de la justice au tribunal $(A m 5,10)$ afin de mieux extorquer des rançons, oppresser et pressurer l'indigent en toute quiétude (Am 5,11-12). Ces actes présentés, en Am 5,10-12, comme la conduite permanente des coupables corroborent ainsi la teneur d'Am 5,7 en ce qu'ils dénotent que le droit et la justice sont complètement bafoués, bouleversés et dénaturés en Israël.

Ensuite, cette structure montre surtout un parallélisme antithétique très accentué ou un contraste abyssal entre les actions de ceux qui bouleversent la mišpa àt et la șadāqāh [AA' (Am 5,7.10-12)] et celles de Dieu situées au centre [B (Am 5,8-9)] et exaltées sous forme d'une doxologie. En effet, le prophète attribue à Dieu, le Seigneur de l'univers, cinq actions qui portent apparemment sur le cosmos et non sur les injustices sociales. C'est d'ailleurs dans le but d'accentuer ce contraste qu'Amos ou le rédacteur utilise le même verbe [hăfak $(\mathrm{Am} 5,7.8)]$ pour décrire la transformation du droit en poison et les changements que le Seigneur effectue habituellement dans l'univers. La plupart des exégètes reconnaissent que

22. Nous pensons qu'il ne peut s'agir que du prophète, le seul qui voue une obéissance inconditionnelle à son Seigneur (Am 3,8) et est pris en aversion tout au long du livre (Am 2,12; 7,10-17). Bovati et Meynet $(1994,171)$ voient également en Am 5,10 le refus du prophète. 
la répétition du verbe hāfakı en Am 5,8 n'est pas fortuite (Wolff 1977, 241; Jeremias 1998, 90-91). Elle est voulue pour marquer une opposition entre les actions de Dieu décrites en Am 5,8-9 et celles des auteurs des injustices énoncées en Am 5,7 puis explicitées en Am 10-13. Cette opposition intentionnelle nous rappelle le premier discours qu'Amos a prononcé contre Israël (Am 2,6-16 $)^{23}$. Sa prise en compte s'avère très éclairante. Elle nous incite à nous interroger sur la signification profonde des actions qu'Amos attribue à Dieu afin de mieux cerner les conséquences de la perversion du droit et de la justice sur le projet créateur et sur la destinée d'Israël. Dès lors, quelle signification revêt chacune des cinq actions que le prophète présente, en Am 5,8-9, comme la conduite permanente de Dieu au sein de la création?

La première action porte sur la création des Pléiades et de l'Orion. Son interprétation ne pose pas de grandes difficultés. La majorité des commentateurs modernes, à la lumière de $\mathrm{Jb} 38,31$, un passage dans lequel les Pléiades et l'Orion désignent des constellations, considèrent ces deux termes comme les noms des deux astres traditionnellement associés à la Nouvelle année (Nisan) ou au changement de l'hiver et de l'été (Fohrer 1963, 216; Hammershaimb 1970, 80-81; Paul 1991, 168). Aussi, en dépeignant Dieu comme celui qui façonne les Pléiades et l'Orion, Amos le présente-t-il, métaphoriquement, comme celui qui est à l'origine du cycle des années et des saisons mais surtout comme celui qui assure leurs successions régulières dans le temps. Les deux actions suivantes concernent respectivement le changement de l'obscurité en clarté matinale et la réduction du jour en sombre nuit; leur explication ne pose pas davantage de problèmes majeurs. Les exégètes, comme Andersen et Freedman (2008, 491), admettent généralement qu'elles rappellent le récit de la création et plus particulièrement, la création de la lumière $(G n$ 1,2-5) et celle des étoiles (Gn 1,16). Ainsi, lues à la lumière de Gn 1,2-5 et de Gn 1,16, ces deux actions dénotent que le Dieu d'Israël assure réellement le changement régulier des jours et des nuits, les deux moments du temps journalier.

Quant à la quatrième action, elle est formulée comme suit: "il convoque les eaux des mers pour les répandre sur la surface de la terre». Son explication suscite deux grandes prises de positions opposées. Certains

23. En Am 2,6-16, le prophète oppose les exactions des coupables (Am 2,6-8.12) aux actes fondateurs d'Israël que Dieu a accomplis. Pour plus de précisions sur une structure qui dévoile ce contraste et son implication pour la lecture d'Am 2,6-12, nous renvoyons le lecteur à notre article qui vient de paraître. 
commentateurs y voient le prélude d'une catastrophe de proportion cosmique et lui donnent deux significations très similaires. D'une part, ils estiment, qu'Amos annonce à ceux qui dénaturent le droit et la justice que Dieu est sur le point d'enclencher le processus inverse de ce qu'il fit respectivement le deuxième et le troisième jours de la création de l'univers, c'est-à-dire, l'établissement du firmament séparant les eaux terrestres des eaux célestes $(\mathrm{Gn} 1,6)$ et les eaux inférieures de la terre ferme (Gn 1,9; Jb 38,8-11; Pr 8,29; Ps 104,9). Autrement dit, comme en Jr 4,23-26, cette action traduirait le retour de la terre au chaos ou du moins à l'aspect qu'elle avait avant l'intervention de Dieu après le premier jour de la création (Bovati et Meynet 1994, 173). D'autre part, en s'appuyant sur le récit du déluge, ils avancent que cette quatrième action prend la connotation d'une annonce d'un déversement des océans sur la terre comme au jour de Noé. Ils estiment également qu'en dépit de l'engagement de Dieu dans Gn 8,21 à ne plus anéantir la création, Amos avertit les coupables de crimes contre la justice qu'à cause de leurs exactions, Dieu est sur le point de provoquer une inondation similaire à celle du temps de Noé afin d'anéantir de nouveau toute la création (Olher 1970, 92-100; Andersen et Freedman 2008, 493). En un mot, pour les partisans de cette ligne d'interprétation, Amos annoncerait la fin du monde: Dieu va défaire la création en ramenant les eaux de la mer sur la terre.

D'autres biblistes, comme Speier (1953, 305-310), Amsler (1982, 211), Mays $(1969,95-96)$ et Story $(1980,72)$, maintiennent qu'à travers le syntagme «il convoque les eaux des mers pour les répandre sur la face de la terre ", le prophète dresse plutôt le portrait d'un Dieu présidant aux cycles des eaux, qui s'évaporent des mers pour retomber en pluie sur la terre. Nous privilégions cette interprétation en posant qu'Amos dépeint Dieu comme celui qui envoie la pluie pour nourrir la terre. Elle a notre préférence parce que, tout d'abord, le verbe qârâ (appeler, convoquer) utilisé pour décrire l'acte de convoquer les eaux des mers, tout comme les verbes 'as̄āh (faire ou façonner) et $h \bar{a} f a \underline{k}$ (changer) $k \bar{a} \check{s} a \underline{k}$ (obscurcir) employés en Am 5,8, décrit une action située dans le présent. Ensuite, le verbe qârâ est au participe présent, temps qui indique habituellement une action continue, en cours. Le temps de ce verbe implique incontestablement que c'est de façon régulière et continuelle que Dieu convoque les eaux pour les répandre sur la terre; il ne s'agit donc pas d'une action ponctuelle qui sera accomplie dans un futur proche ou lointain. Enfin, cette interprétation corrobore certaines données du livre. Elle dévoile qu'en tant que maître des pluies et des saisons, Dieu seul peut provoquer 
la sécheresse $(\mathrm{Am} 4,7)$ ou le dessèchement des pâturages et de la crête du Carmel (Am 1,2).

La cinquième action est énoncée comme suit: «il fait surgir la dévastation sur l'homme fort au pillage et la dévastation entre dans la citadelle». Beaucoup d'exégètes éprouvent des difficultés à établir le lien entre elle et celles exposées dans Am 5,8. Certains considèrent Am 5,9 comme une interpolation tardive et complexe provenant d'un autre contexte, probablement d'Am 6,14 (Watts, 1956, 33-39) ou d'Am 4,13 (Wolff 1977, 241; Soggin 1987, 89-92). Mais en fait, son explication dépend essentiellement du sens que les uns et les autres accordent au terme hammavali $\bar{g}$, participe présent du verbe bālag au hifil. Par exemple, van Hoonacker $(1908,241)$, qui privilégie le sens étymologique de ce terme, interprète cette action comme suit: «Dieu fait briller le salut pour le malheureux et sur la forteresse il amène la destruction ». Mais, dans la traduction d'Am 5,7-13 (voir note 17), nous nous sommes inscrit dans la perspective des commentateurs qui rendent ce verbe par «fait surgir». Nous maintenons donc qu'en Am 5,9 , le prophète annonce que seul Dieu fait surgir la dévastation sur l'homme fort.

Pour appréhender la signification profonde de la cinquième action qu'Amos attribue à Dieu, il convient de se poser la question suivante: qu'est-ce que la description de Dieu comme l'unique puissant capable de livrer l'homme fort à la dévastation pour que celle-ci entre dans la citadelle implique pour la sécurité et la survie d'Israël? Cette interrogation nous permet de déduire qu'en exaltant le Dieu d'Israël comme le seul qui soit en mesure de livrer l'homme fort au ravage afin que celui-ci pénètre dans la ville, Amos insinue que si la citadelle — c'est-à-dire la Samarie (la capitale d'Israël) dans laquelle l'on bouleverse la justice — n'a pas encore connu de dévastation, c'est que le Seigneur a toujours épargné ses hommes forts chargés d'assurer sa défense. Par conséquent, sans le Seigneur, l'ennemi serait déjà entré pour semer le chaos dans la Samarie, la ville fortifiée du Royaume du Nord. En définitive, l'énoncé d'Am 5,9 dresse le portrait d'un Dieu qui assure de façon permanente la sécurité et la paix en Israël et, audelà, dans l'univers.

De l'explication des cinq actions énumérées en Am 5,8-9, nous pouvons déduire que Dieu effectue le changement régulier des temps et des saisons dans le but du maintien de l'ordre des choses dans le règne de la création et assure la paix et la stabilité d'Israël (Carroll 2002, 229). En tant que créateur et maître de l'univers, Dieu assure constamment le rythme régulier des temps et des saisons pour maintenir en vie ses créatures et garantit la 
sécurité, la paix dans le règne de la création. Or, quelques-uns parmi les fils d'Israël pervertissent quotidiennement le droit et la justice, deux valeurs essentielles pour garder l'équilibre et l'harmonie au sein d'une société et, au-delà, de l'univers. En dénaturant ces deux valeurs essentielles, ils détruisent donc les fondements d'un univers juste et harmonieux que Dieu s'attèle à maintenir et instaurent ainsi le désordre ou le chaos social. C'est assurément cet effondrement inéluctable d'Israël que le prophète, l'homme sage et avisé mais haït et exécré $($ Am 5,10), perçoit et il se tait $(A m 5,13)$.

Dès lors, nous comprenons pourquoi Amos présente les crimes contre la justice comme des peša 'îm (Am 2,6; 3,14; 5,12), c'est-à-dire, des actes de rébellion contre Dieu créateur. En effet, en instaurant le désordre dans le règne de sa création, les auteurs des exactions énumérées en Am 5,7.1012 et de celles dénoncées dans les autres oracles du livre (Am 2,6-8; 3,9$10 ; 4,1 ; 6,4-6 ; 8,4-6)$ s'opposent à Dieu ou entrent en sédition contre Celui qui s'attelle constamment à maintenir un ordre juste et harmonieux dans l'univers. En conséquence, toute situation d'injustice devient indubitablement une atteinte au projet créateur parce qu'elle instaure la confusion au sein de la création. En définitive, Am 5,7-13 révèle que les injustices sont des actes de "dé-création» (Bovati et Meynet 1994, 173). Elles font retourner la société au chaos.

L'analyse d'Am 5,7-13 nous donne à voir que les injustices sont des facteurs de ruine, qui entraînent l'anéantissement des coupables avec tout ce qu'ils ont acquis et bâti (Am 3,15; 5,11). Il résulte de cette observation que toute société dont les habitants canonisent les injustices comme moyens de gain, s'engage dans un processus de désagrégation. C'est d'ailleurs la raison pour laquelle Amos pleure la ruine d'Israël comme si elle avait déjà eu lieu (Am 5,1-3) ou présente sa chute comme imparable (Am 5,16-20). C'est aussi pourquoi il invite de façon pressante les auteurs d'injustices à rechercher Dieu en rétablissant la justice au tribunal afin de vivre (5,4.14-15). Infestée d'injustices, Israël apparaît à ses yeux comme une société déjà morte (Am 4,1-3; 5,16;8,3). C’est encore pourquoi Amos affirme - contrairement aux autorités politiques et aux fortunés qui se croient en paix et appellent de tous leurs vœux la venue du jour du Seigneur (Am 5,18; 6,1.3) - que l'homme avisé se tait (Am 7,13); lui, perçoit un avenir très sombre (Am 5,16-20;6,3.7-11). C'est enfin pourquoi, plus loin, il incite instamment les coupables à faire jaillir le droit comme de l'eau et la justice comme un torrent intarissable (Am 5,24). L'une des maximes à tirer de la critique sociale d'Amos, laquelle a justement déjà été relevée sous une autre forme par Bons, est la suivante: Israël et, par-delà, 
aucune société ne peut survivre sans que l'agir de ses habitants soit commandé par deux valeurs essentielles, à savoir, le droit et la justice ${ }^{24}$. Aussi, comme les coupables d'Am 5,7-13, toutes les personnes qui, dans leurs choix politiques et économiques, ne se laissent pas guider par ces deux valeurs mais les bafouent pour s'enrichir, tirent leur société vers un chaos de dimension cosmique. Ce message, qui trouve un très large écho et son prolongement dans la prédication d'autres prophètes successeurs d'Amos - tels que Osée (Os 8,7), Agée (Ag 1,6), Sophonie (So 1,13) et surtout Isaïe (Is 5,11-3) et Michée (Mi 3,1-4; 6,15) —, laisse présumer que les acteurs d'injustices seraient en réalité comparables à des insensés, posant des actes qui entraîneront leur propre destruction (Am 5,11.16-20). Cette dimension du message d'Amos sera mise en exergue dans l'analyse d'Am 6,12 que nous présentons dans les lignes suivantes.

\section{La perversion du droit et de la justice, acte insensé et autodestructeur} $($ Am 6, 12)

Am 6,12 est également un passage clef du livre d'Amos. Il est notamment l'un des éléments essentiels du chapitre six qui est un discours de malheur dans lequel le prophète dénonce vivement l'orgueil et l'insouciance des autorités politiques et militaires (Am 6,8), et l'attitude des riches qui se vautrent dans un luxe exubérant, passent leur temps à organiser de monstrueux festins dans leurs palais situés sur la montagne de Sion sans se préoccuper de «la ruine de Joseph» (Am 6,6). Beaucoup de commentateurs le considèrent comme un aphorisme sapientiel (Amsler 1982, 221; Vesco 1980, 498; Soggin 1987, 110). En effet, sa tonalité interrogative et son contenu le distinguent nettement des autres éléments d'Am 6,1-14, plus particulièrement, d'Am 6,8-11 et d'Am 6,13-14 qui constituent respectivement son contexte antérieur et postérieur immédiat. Il s'articule en deux parties dont la première (Am 6,12a) comprend une double question rhétorique présentant chacune une action absurde et impossible, action qu'aucune personne en pleine possession de ses facultés mentales ne saurait songer à accomplir: Hayrușūn bassela sūsîm (Les chevaux peuvent-ils galoper sur les roches ?) et 'im yaћărōwōš babbaqārîm (peut-on labourer la mer avec des boufs). La seconde (Am 6,12b) consiste également en une ques-

24. Bons souligne que le fondement éthique du livre d'Amos réside dans sa conviction que l'agir des israélites doit être commandé par la mišpāt et la șadāqāh (Bons 2012, 107). 
tion dont le contenu rappelle la dénonciation de la perversion de la mišpāt

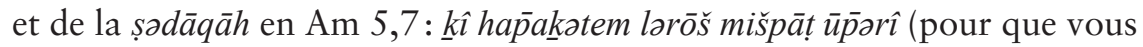
changez le droit en ciguë25) et șadāqāh lala ăănăh (et la justice en poison).

L'articulation de ce passage laisse entrevoir la stratégie rhétorique très remarquable adoptée par Amos. La première double question (Am 6,12a) fonctionne en fait comme une parabole dont la visée est de permettre à ceux qui changent continuellement la nature du droit et de la justice (Am 6,12b) de déceler le caractère totalement incompréhensible et insensé de leur comportement (Martin-Achard 1990, 77). Le prophète présente en Am 6,12a deux exemples d'actes absurdes pour aussitôt les appliquer explicitement à des crimes contre la justice qu'il énumère en Am 6,12b. En procédant ainsi, il invite tout d'abord ceux qui changent la nature du droit et de la justice à comparer leur état d'esprit avec celui d'une personne qui oserait faire galoper ses chevaux sur les rochers ou atteler ses bœufs pour labourer la mer (Am 6,12). Ensuite, il les incite à établir une analogie entre le sort de leur nation, Israël, et celui des chevaux et des bœufs que l'on obligerait respectivement à courir sur un terrain rocailleux et à labourer la mer. Les deux implications majeures de la stratégie rhétorique d'Am 6,12 que nous venons de dégager nous permettent de tirer aisément deux grands enseignements concernant respectivement l'état d'esprit de ceux qui commettent les injustices sociales et la destinée de la société dans laquelle ils vivent.

À la lumière des observations qui précèdent, l'un des enseignements que nous pouvons tirer d'Am 6,12 est celui déjà relevé par Bovati et Meynet (1994, 219). Ces auteurs soulignent que la pédagogie d'Amos vise à faire comprendre à ceux qui bouleversent le droit et la justice qu'ils «sont assez stupides pour les changer en poison et en absinthe, pour en faire des instruments de mort. Leur manque d'intelligence les empêche de voir que ce ne sont pas seulement les pauvres qu'ils empoisonnent, mais qu'ils se condamnent eux-mêmes à mort en ne pratiquant pas la justice ». Autrement dit, comme aucune personne sensée n'oserait faire galoper ses chevaux sur des rochers en prenant le risque de perdre ses animaux mais aussi ses chars et sa vie elle-même, ceux qui commettent les crimes contre la justice, dénoncés en Am 6,12b et dans tous les autres oracles du livre d'Amos (Am $2,6-8 ; 3,9-10 ; 4,1 ; 5,7.10-12 ; 8,4-6)$, font preuve d'un manque manifeste

25. Larōš, rendu par "ciguë", est souvent employé comme synonyme de la ănāh (Dt 29,17). Il désigne également une plante toxique ou venimeuse (Dt 32,32), du poison $(\mathrm{Jr} 8,14)$ et encore du venin (Dt 32,33). 
de bon sens. En bouleversant le droit et la justice pourtant indispensables pour la paix et la stabilité sociale, ils prennent le risque de saper les fondements de leur société et de l'entraîner ainsi vers une ruine imparable, qui les emportera eux aussi avec tout ce qu'ils ont injustement acquis. En définitive, il résulte que, pour Amos, toute personne qui pratique les injustices sociales instille du poison mortel dans son propre univers de vie, un poison qui finira par l'atteindre lui-même. Par conséquent, tous ceux qui bafouent la justice et le droit sont des personnes insensées et déraisonnables.

L'autre enseignement majeur que nous retenons d'Am 6,12 va dans le même sens. Il concerne davantage la destinée d'Israël et, par-delà, celui de toute société dont une partie des habitants font de l'injustice et de la paupérisation des moyens licites pour accroître leur fortune. En effet, en appliquant les deux situations absurdes d'Am 6,12a à la perversion de la justice qui prévaut à Samarie (Am 6,12b), le prophète insinue que tout ordre est renversé dans cette nation et qu'elle ne peut donc plus, dans cet état de choses, poursuivre sa destinée. Aussi, Amos, en comparant la situation de la Samarie à celle des chevaux ou des bœufs que leurs propriétaires respectifs font courir sur les rochers et labourer la mer, laisse-t-il entendre qu'Israël va à sa ruine. Dans cette même perspective, van Hoonacker (1908, 260) souligne qu'en Am 6,12, "le prophète donne deux exemples de choses impraticables, pour insinuer que l'état de choses analogue [sic] qui règne à Samarie est contraire à toute raison et ne peut pas durer plus longtemps ». Tel le cheval que son propriétaire fait galoper sur les rochers est irréversiblement condamné à une chute, Israël dont les fondements, la mišpāt et șadāqāh, sont sapés, va vers un écroulement fatal. Ce message renforce celui d'Am 5,7-12, dévoile tout le génie rhétorique de ce prophète et montre qu'il ne s'est pas contenté de critiquer de façon acerbe l'opulence et le luxe exacerbés des riches de son temps. Il apparaît comme un homme éclairé qui, sans se laisser intimider et corrompre, a su dans une société où les injustices devenaient licites, affirmer que toute société qui se bâtit sur des injustices graves est une société qui marche à l'envers et ne peut plus poursuivre sa route.

\section{Conclusion}

Nous pouvons récapituler les résultats de cette étude à travers les observations suivantes. L'analyse du contexte du ministère d'Amos nous a permis de mettre en exergue les motifs de son intervention dans le Royaume du 
Nord. Nous avons retenu que ce prophète n'a pas été envoyé pour féliciter les autorités politiques, militaires et religieuses d'avoir agrandi les frontières d'Israël et fait d'elle une nation paisible et prospère. Sa mission, telle qu'elle apparaît dans les deux passages étudiés (Am 5,7-13; 6,12), a plutôt consisté à attirer leur attention sur le fait que les graves injustices sociales qu'elles légitiment en vue d'accroître leurs richesses mettent sérieusement en péril l'avenir de la nation et le leur, sans qu'elles ne s'en rendent compte. Ainsi, sans se compromettre en se laissant corrompre, il a passé au crible toutes les structures sociales d'Israël afin de leur montrer que la mišpāt et la şadāqāh, les deux piliers sur lesquels la société devrait reposer sont complètement sapés et que, par conséquent, celle-ci est condamnée à s'écrouler fatalement si elles ne se détournent pas de leurs attitudes. L'une des formes les plus perverses des injustices qu'il a dénoncées est celle commise dans les instances même de la justice et par les hommes chargés de la rendre. Cette forme d'injustice est beaucoup plus subtile et perfide parce qu'elle justifie et canonise les injustices économiques et conduit à une catastrophe totale, un chaos de dimension cosmique (Am 5,7-8; 8,8; 9,5).

Nous en déduisons également que, pour permettre à ceux qui bouleversent la mišpāt et la șadāqāh de prendre conscience que leurs comportements ébranlent sérieusement les fondements de base de leur nation et mettent en péril sa destinée, Amos ne s'est pas contenté de critiquer leur richesse et leur mode de vie frivole. Il a articulé son message de manière à leur faire comprendre que toute situation d'injustice est contraire au projet de Dieu pour les humains et demeure, par conséquent, un facteur de ruine et de dé-création. L'analyse d'Am 5,7-13 nous a permis de montrer cette dimension essentielle de sa critique sociale. Nous y avons principalement relevé qu'il oppose le comportement habituel des auteurs d'injustice à l'action permanente de Dieu qui vise à assurer la vie, l'ordre, l'harmonie, la paix et la sécurité au sein de la création. Ce contraste nous a permis d'établir qu'Amos annonce à ceux qui pervertissent le droit et la justice qu'ils renversent l'ordre juste et harmonieux que Dieu s'attèle à maintenir et tirent, de ce fait, leur société vers un chaos qui les emportera avec tous leurs biens (Am 5,11). Dès lors, nous avons dégagé qu'Amos entendait dire à ses contemporains qu'une société qui néglige le droit et la justice et se bâtit sur la paupérisation, est une société dont les fondements sont ruinés, une société vouée à s'écrouler.

Cette maxime nous a conduit à étudier Am 6,12 pour montrer qu'Amos présente en fin de compte les injustices comme des actes insensés et absurdes. Il ressort de cette analyse que, pour Amos, toute personne qui 
commet des injustices sape l'harmonie et la stabilité et, par là-même, entre en rébellion contre Dieu créateur. De même, cette personne manque de bon sens parce qu'elle prend le risque d'instiller du poison dans son propre milieu de vie ou de tirer sa société vers un précipice ou encore de poser les fondations de sa nation sur une bombe à retardement. Ceux qui font des injustices sociales un moyen d'accroître leur richesse sont des insensés parce qu'ils amassent des biens dont ils ne profiteront point (Am 3,15; 5,10-11).

Dans cette perspective, même si Amos est intervenu dans un contexte précis, son message vaut tout de même pour tous les temps et surtout pour notre monde d'aujourd'hui où les grandes multinationales trouvent des moyens de contourner les lois pour accroître leur capital au détriment des pauvres qui se sentent de plus en plus asphyxiés. Son message est pertinent et d'actualité parce qu'il nous enseigne que la justice et le droit sont le pivot sur lequel gravite la destinée de toute structure sociale; leur perversion entraîne une décadence sociale, une ruine imparable qu'aucune stratégie ne peut arrêter. Dans un monde où la lutte sociale se pose de plus en plus avec acuité, Amos nous donne les outils nécessaires pour pouvoir dire à nos contemporains que toute société dont les habitants légitiment les injustices pour s'enrichir est une société qui marche à l'envers, une société dont la durée de vie est comptée. Il nous apprend également que toute société qui se bâtit sur des injustices graves est déconnectée de Dieu sans qui rien ne peut survire et réciproquement qu'une telle déconnexion conduit à l'exploitation de l'homme, qui entraîne un effondrement social irréversible.

\section{Références}

Amsler, S. (1982²) [1969], "Amos", dans E. Jacob, C.-A. Keller et A. Amsler, dir., Osée, Joël, Amos, Abdias, Jonas, Genève, Labor et Fides (Commentaire de l'Ancien Testament 11a), p. 157-247.

(1990), L’homme de Teqoa. Message et commentaire du livre d'Amos, Aubonne, éditions du Moulin.

Andersen, F. et D. N. Freedman (2008) [1989], Amos. A New Translation with Introduction and Commentary, New Haven/London, Yale University Press (Anchor Bible 24A).

Asurmendi, J. (1988), Amos et Osée, Paris, Cerf (Cahiers Évangile 64).

Bons, E. (2012), "Amos et la contestation des pouvoirs ", dans D. LuCiani et A. Wenin, dir., Le pouvoir. Enquêtes dans l'un et l'autre Testament, Paris, Cerf (Lectio Divina 248), p. 95-110. 
Botterweck, G. J. (1974), «'ebhyon», dans G. J. BotTerweck, et al., dir, Theological Dictionary of the Old Testament, vol.1 / trad. par D. W. Stott, Grand Rapids, Eerdmans, p. 27-41.

Bovati, P. et R. Meynet (1994), Le livre du prophète Amos, Paris, Cerf.

Carroll, R. (2002), Amos. The Prophet and His Oracles, Louisville, Londres.

Hahling, C. (2013), Vie avec Dieu et injustice sociale. Le prophète Amos et nous, Romanel-sur-Lausanne, Scripsi.

Hasel, G. F. (1991), Understanding the Book of Amos. Basic Issues in Current Interpretation, Grand Rapids, Baker Book House.

Hoonacker, A. van (1908), Les douze petits prophètes, Paris, Librairie Victor Lecoffre, J. Gabalda et Cie.

Jeremias, J. (1998) [allemand 1995], The Book of Amos. A Commentary / trad. par W. D. Stott, Louisville, Kentucky, Westminster John Knox Press (Old Testament Library 2).

Kolani, N. B. (2016), «Amos, prophète et défenseur de la dignité humaine. Une lecture d'Am 2,6-16 à la lumière de la Déclaration universelle des droits de l'homme", dans P. KeITH, dir., Texture sacrée, l'intertexte biblique dans quelques ouvres littéraires et textes autorisés, Strasbourg, Presses Universitaires de Strasbourg, p. 59-92.

Lemaire, A. (1981), Histoire du peuple hébreu, Paris, Presse Universitaire de France (Que sais-je? 1898).

Loretz, O. (1975), «Die prophetische Kritik des Rentenkapitalismus. Grundlagen-Propbleme der Prophetenforschung », Ugarit-Forschungen, 7, p. 271-278.

Martin-Achard, R. (1984), Amos, l'homme, le message, l'influence, Genève, Labor et Fides.

Mays, J. L. (1969), Amos. A Commentary, Philadelphia, Westminster Press (Old Testament Library).

Paul, S. (1991), Amos. A Commentary on the Book of Amos / édité par F. M. Cross, Philadephie, Fortress Press (Hermeneia).

Pfeiffer, G. (1991), "Jahwe als Schöpfer der Welt und Herr Ihrer Mächte in der Verkundigung des Propheten Amos», Vetus Testamentum, 41, p. 475-481.

Renan, E. (1889), Histoire du peuple d'Israël, Tome 2, Paris, Calmann Levy. 
Schotтroff, W. (1984) [allemand 1979], God of the Lowly. Socio-historical Interpretations of the Bible / éd. par W. Schottroff et W. Stegemann, New York, Orbis Books.

Silva, A. da (1997), Amos, un prophète "politiquement incorrect», Montréal, Médiaspaul (Parole d'actualité 7).

Soggin, J. A. (1987) [Italien 1982], The prophet Amos / trad. par J. Bowden, London, SCM.

Speier, S. (1953) «Bemerkungen zu Amos », Vetus Testamentum, 3, p. 305310.

Story, C. I. K. (1980), «Amos-Prophet of Praise», Vetus Testamentum, 30, p. 67-80.

Vesco, J.-L. (1980), "Amos de Teqoa, défenseur de l'homme", Revue Biblique, 4, p. 481-513.

WaArd, J. de (1977), "The Chiastic Structure of Amos V 1-17», Vetus Testamentum, 27, p. 170-177.

Wanke, G. (1972), "Zur Grundlagen und Absicht prophetischer Sozialkritik », Kerygma und Dogma, Göttingen, 18, p. 2-17.

WolfF, W. (1977) [allemand 1975 (1969)], Joel and Amos. A Commentary on the Book of Joel and Amos / trad. par W. Janzen, S. D. McBride, Jr. et C. A. Muenchow, Philadelphie, Fortress Press (Hermeneia).

\section{Résumé}

De nos jours, la poussée des mouvements extrémistes et des violences liées à la précarité et aux inégalités pose avec plus d'acuité la question de la justice sociale. Il importe que les chrétiens et tous les hommes militant pour un avenir paisible, réentendent la voix des prophètes bibliques, lesquels ont dénoncé et présenté les injustices sociales comme facteurs de ruine. Dans cette perspective, il est urgent de réécouter plus particulièrement la voix d'Amos qui, dans l'histoire de la religion biblique, a su démontrer, pour la première fois, que les injustices sociales sont des actes insensés et autodestructeurs. Bien que proclamé il y a plus de 2500 ans, son message est d'actualité pour notre monde marqué par des tractations entre des débiteurs essoufflés des créanciers impitoyables vivant dans la surabondance; il nous rappelle que toute société infestée par des injustices et de profondes inégalités sociales s'engage dans un processus de dé-création. 


\section{Abstract}

Today, the thrust of extremist movements and violence linked to insecurity and inequality shows the question of social justice under sharp acuteness. It is important that Christians and all men campaigning for a peaceful future hear biblical prophets' voice once more. These prophets have denounced social injustices and pointed them out as ruin factors. In this perspective, Amos' voice must to be listened urgently and more specifically. In the history of biblical religion, he was the first to make clear the social injustices are senseless and self-harming. Although urging a change of life, more than 2500 years ago, Amos' message is still relevant in our current world. It's marked by dealings between unscrupulous debtors and ruthless creditors who live in superabundance. So, Amos reminds us that any society in this world, being infested by injustices and deep social inequalities, has to commit oneself in a de-creation process. 